\title{
Histoire Politique
}

\section{Samuel Boussion, Mathias Gardet, Martine Ruchat, L'Internationale des républiques d'enfants, 1939-1955}

Paris, Anamosa, 2020, 479 p.

\section{Camille Mahé}

\section{(2) OpenEdition}

\section{Journals}

Édition électronique

URL : https://journals.openedition.org/histoirepolitique/744

DOI : 10.4000/histoirepolitique.744

ISSN : 1954-3670

Éditeur

Centre d'histoire de Sciences Po

Référence électronique

Camille Mahé, « Samuel Boussion, Mathias Gardet, Martine Ruchat, L'Internationale des républiques d'enfants, 1939-1955», Histoire Politique [En ligne], Comptes rendus, mis en ligne le 19 avril 2021 , consulté le 21 octobre 2021. URL : http://journals.openedition.org/histoirepolitique/744 ; DOI : https:// doi.org/10.4000/histoirepolitique.744

Ce document a été généré automatiquement le 21 octobre 2021.

Histoire Politique 


\section{Samuel Boussion, Mathias Gardet, Martine Ruchat, L'Internationale des républiques d'enfants, 1939-1955}

Paris, Anamosa, 2020, 479 p.

Camille Mahé

\section{RÉFÉRENCE}

Samuel Boussion, Mathias Gardet, Martine Ruchat, L'Internationale des républiques d'enfants, 1939-1955, Paris, Anamosa, 2020, 479 p. 
1 Au cours de l'été 1948 , soit près de trois ans après la fin des hostilités, une petite quarantaine de délégués, observateurs et experts internationaux spécialistes de l'enfance se réunit en Suisse sous l'impulsion de la toute jeune Organisation des Nations unies pour l'éducation, la science et la culture (Unesco). Ils se retrouvent plus précisément à Trogen, dans le village d'enfants Pestalozzi. Fondé en 1945 pour accueillir de jeunes orphelins victimes de la guerre issus de nationalités différentes, le village constitue une initiative originale, presque utopique, dans une Europe en ruines. Projet humanitaire et surtout pédagogique, il se veut un havre de paix pour (re)construire l'entente et l'amitié entre les peuples afin d'asseoir la démocratie pour les décennies à venir. Face à la multiplication de

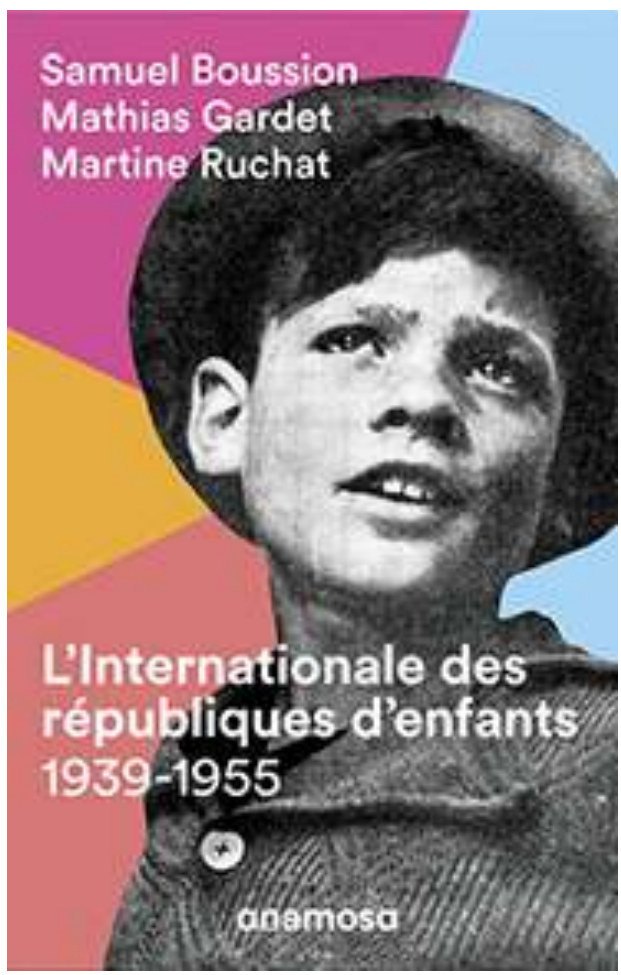
« communautés », «villages» ou encore « républiques» d'enfants à travers l'Europe - on en dénombrerait une trentaine en 1948 -, l'objectif de la conférence est de définir et surtout de poser un cadre commun à ces initiatives. Elle est toutefois le point d'orgue d'une expérience éphémère, puisque cette "internationale des républiques d'enfants " ne survit pas à la Guerre froide. Tombée dans l'oubli, elle est restée dans l'ombre, jusqu'à ce que le travail collectif mené depuis une dizaine d'années par Samuel Boussion, Mathias Gardet et Martine Ruchat fasse de nouveau lumière sur elle.

2 Le format adopté par ces chercheurs en histoire, sciences de l'éducation et psychologie est original et hybride. Chacun des douze chapitres se voit précédé d'un résumé et d'une courte biographie des femmes et des hommes qui ont joué un rôle central dans la thématique abordée. Cela en fait un outil particulièrement utile pour qui cherche à identifier non seulement celles et ceux qui prirent part aux expériences des républiques d'enfants, mais aussi les nombreux acteurs qui contribuèrent à diffuser les idées de l'éducation nouvelle, de la psychologie et de la psychiatrie infantile au lendemain de la Seconde Guerre mondiale. Le lecteur a également la possibilité de se rendre sur le carnet de recherche publié en parallèle sur internet pour suivre les travaux des auteurs ${ }^{1}$. Surtout, ces derniers ont pris le parti d'adopter « une écriture de l'histoire qui soit la plus narrative possible, sans trop de cassures de rythme ${ }^{2}$ ». Si ce choix peut parfois frustrer le lecteur dans la mesure où le livre est dénué de notes de bas de pages et de fin de chapitre ${ }^{3}$, il aura certainement le mérite d'attirer le grand public. Enfin, le foisonnement d'illustrations - lesquelles gagneraient peut-être parfois à être davantage intégrées au propos des auteurs - participe à rendre vivante l'histoire de ces républiques d'enfants.

3 Pour mener à bien leur projet, les auteurs se sont appuyés sur des sources riches et nombreuses, issues de fonds d'organisations internationales - notamment celles de 
l'Union internationale de secours aux enfants (UISE), de l'Unesco, du Bureau international d'éducation (BIE), de l'œuuvre de secours aux enfants (OSE)-, d'archives privées (parmi lesquelles celles de Robert Préaut ou de Ernesto Codignola) et de la documentation conservée par les communautés d'enfants, principalement françaises, italiennes et suisses. On notera que seule la voix des premiers concernés manque, quoiqu'elle apparaisse ponctuellement dans certains chapitres. Le tort n'en revient pas aux auteurs, qui ont été «frappés par le fait que la parole des jeunes passés par ces républiques d'enfants reste finalement peu audible» dans les archives ${ }^{4}$. Peut-être qu'une enquête orale (quoique difficile dans la mesure où les témoins ont probablement disparu pour nombre d'entre eux) ou la recherche de témoignages écrits permettraient de combler ce vide. Une recherche sur internet révèle en effet qu'à l'échelle locale, des événements mémoriels entretiennent le souvenir de ces communautés ${ }^{5}$.

La trame du livre suit une logique chronologique ternaire axée autour de la conférence de Trogen : avant, pendant et après. Au cours des cinq premiers chapitres, les auteurs reviennent sur quelques initiatives nées indépendamment du village Pestalozzi et sans véritable concertation entre elles, essentiellement en France, en Italie et en Suisse. Les deux premiers chapitres rappellent d'abord que les racines des républiques d'enfants sont plus profondes qu'elles n'y paraissent : elles sont à chercher du côté des États-Unis à travers le modèle de la George Junior Republic créée en 1895 par le protestant William Reuben Georges et la Boys Town fondée en 1917 dans le Michigan par le père irlandais catholique Edward Joseph Flanagan. Surtout, la première véritable communauté d'enfants est née avant la Seconde Guerre mondiale, au moment de la guerre civile espagnole. Soucieuse de venir en aide aux réfugiés qui ont traversé la frontière pyrénéenne, l'Union internationale de secours aux enfants fut l'un des principaux acteurs de sa mise en place, tout en s'appuyant sur un réseau d'acteurs de l'aide nés après la Grande Guerre. De fait, les communautés d'enfants sont d'abord le reflet d'une initiative humanitaire (sinon philanthropique), avant d'être gagnées par des objectifs pédagogiques.

5 Les cinq premiers chapitres dévoilent, par la finesse des détails exposés quant à la naissance de tels projets, à quel point la création de villages, communautés et autres républiques fut avant tout le fait d'initiatives personnelles. Il aura fallu compter entre autres sur la pugnacité d'Henri Julien et Henriette Pauriol-Julien pour construire la Libre République de Moulin-Vieux dans les Alpes françaises, de Antonio Rivolta pour la Repubblica dei Ragazzi de Civitavecchia près de Rome, de Walter Robert Corti, Marie Meierhofer et Elisabeth Rotten pour le Kinderdorf Pestalozzi, de Robert Préaut pour le Hameau-École d'Île-de-France, ou encore de Ernesto Codignola pour la Scuola-Città de Florence. L'histoire de l'internationale d'enfants est aussi une histoire de difficultés financières, matérielles, de désillusions et d'abandons. En ce sens, l'ouvrage est particulièrement riche d'enseignements quant à la mise en place de l'aide à l'enfance victime de la guerre au lendemain de la Seconde Guerre mondiale, davantage étudiée sous l'angle des réalisations plutôt que des échecs.

6 C'est à partir du chapitre 6 que le lien entre toutes ces initiatives émerge pour le lecteur - car les acteurs de cette histoire n'ont jamais été totalement isolés les uns des autres. Ils se sont réunis à de nombreuses reprises, majoritairement en Suisse, pour échanger mais aussi se former afin de diffuser autant que possible le modèle des républiques d'enfants. Tous s'inspirent de l'éducation nouvelle et prônent la participation active des plus jeunes à travers le self-governement : pour pouvoir devenir 
les honnêtes citoyens et travailleurs d'un monde en paix, les enfants doivent eux aussi participer à la construction de leur logement, apprendre un métier, voter, se réunir, élire des gouvernements et des tribunaux. Toutefois, la réalité rattrape dans bien des cas les fondateurs de ces projets ambitieux. Les enfants victimes de la guerre sont davantage encadrés par les adultes qu'autonomes et les communautés sont principalement coupées du reste de la société dans laquelle ses membres doivent pourtant apprendre à vivre. Les tentatives de camps internationaux et de création de structures associatives à la fin des années 1940 sont alors l'illustration de la fragilité de ces initiatives qui ne parviennent pas à trouver une dynamique commune et « s'avèrent être un feu de paille ${ }^{6} »$. Très vite, les critiques internes et externes se multiplient et les priorités nationales prennent le dessus. Certains pays comme la Pologne vont alors jusqu'à rapatrier les enfants qui avaient été installés à Trogen, soulignant combien ils constituent un enjeu politique central dans les sociétés d'après-guerre.

7 Ainsi, l'ouvrage de Samuel Bussion, Mathias Gardet et Martine Ruchat permet de mieux comprendre la période de transition qu'est la sortie de la Seconde Guerre mondiale, où sont nés de nombreux projets, parfois utopiques, pour relever les sociétés en ruines. Il met en évidence le fait que les projets de reconstruction, de rééducation, voire de réhabilitation ne se sont pas arrêtées aux frontières des pays : bien plus, ils s'inscrivent dans une échelle éminemment transnationale, dans la mesure où penseurs, experts, mais aussi et surtout représentants des différents gouvernements échangeaient activement. Les périodes de guerre et de sortie de guerre constituent de fait des " temps transnationaux par excellence ${ }^{7}$ ", comme le rappellent à juste titre les auteurs.

\section{NOTES}

1. https://repenf.hypotheses.org/ [lien consulté le 02/04/2021].

2. Samuel Boussion, Mathias Gardet, Martine Ruchat, L'Internationale des républiques d'enfants, 1939-1955, Paris, Anamosa, 2020, p. 20.

3. Les archives et une bibliographie plus ou moins succincte sont néanmoins brièvement présentées en fin d'ouvrage pour chaque chapitre.

4. Samuel Boussion, Mathias Gardet, Martine Ruchat, op. cit., p. 16.

5. Par exemple: http://lalpedugrandserre.over-blog.com/2016/06/les-70-ans-de-la-republiquede-moulin-vieux.html [lien consulté le 02/04/2021].

6. Samuel Boussion, Mathias Gardet, Martine Ruchat, op. cit., p. 298.

7. Ibid., p. 18. 